\title{
Time-course behavioral features are correlated with Parkinson's disease-associated pathology in a 6-hydroxydopamine hemiparkinsonian rat model
}

\author{
RUI-JUN SU ${ }^{1-3}$, JUN-LI ZHEN ${ }^{1-3}$, WEI WANG ${ }^{1-3}$, JIAN-LIANG ZHANG $^{1,2}$, \\ YAN ZHENG $^{2-4}$ and XIAO-MIN WANG ${ }^{1-3}$
}

\begin{abstract}
${ }^{1}$ Department of Neurobiology, School of Basic Medical Sciences; ${ }^{2}$ Key Laboratory for Neurodegenerative Disorders of The Ministry of Education; ${ }^{3}$ Beijing Institute for Brain Disorders; ${ }^{4}$ Department of Physiology, School of Basic Medical Sciences, Capital Medical University, Beijing 100069, P.R. China
\end{abstract}

Received September 12, 2016; Accepted July 17, 2017

DOI: $10.3892 / \mathrm{mmr} .2017 .8277$

\begin{abstract}
Parkinson's disease (PD) is one of the most common neurodegenerative diseases. For decades, the unilateral 6-hydroxydopamine (6-OHDA) rat model has been employed to investigate the pathogenesis and therapy of PD. However, the behavior and associated pathological features of the model long term have not previously been described dynamically. In the present study, the unilateral model was established by 6-OHDA injection in the striatum. The PD rat model was determined 2 weeks following surgery, according to the apomorphine (APO)-induced rotations, cylinder, rotarod and open field tests. TH-positive neurons and fibers in the substantia nigra pars compacta (SNpc) and striatum, respectively, and glial activation in the SNpc, determined by glial fibrillary acidic protein (GFAP) expression for astrocytes and CD11b (Mac1) expression for microglia, were detected by immunohistological staining. Correlation analysis was performed to understand the association between PD-associated behavior and pathology. The behavioral impairment progressively deteriorated during the process of experiment. In addition, the decrease in TH-positive neurons was associated with an increase in GFAPand Mac1-positive cells in the SNpc. Linear regression analysis indicated the association between behavioral and pathological changes. The results of the present study indicate that the
\end{abstract}

Correspondence to: Professor Yan Zheng, Department of Physiology, School of Basic Medical Sciences, Capital Medical University, 10 Xitoutiao, Youanmen, Fengtai, Beijing 100069, P.R. China

E-mail: zhengyan@ccmu.edu.cn

Professor Xiao-Min Wang, Department of Neurobiology, School of Basic Medical Sciences, Capital Medical University, 10 Xitoutiao, Youanmen, Fengtai, Beijing 100069, P.R. China

E-mail: xmwang@ccmu.edu.cn

Key words: Parkinson's disease, 6-hydroxydopamine, animal model, movement behavior
APO-induced rotation, cylinder and rotarod tests are all sensitive and reliable strategies to predict the loss of $\mathrm{TH}^{+}$neurons. These results provide a potential intervention time-point and a comprehensive evaluation index system for assessment of PD therapeutic strategies using the hemiparkinsonian rat.

\section{Introduction}

Parkinson's disease (PD) is the second most common neurodegenerative disease, and affects $\sim 1 \%$ of individuals $>65$ years of age worldwide (1). It is associated with dopamine (DA) depletion in the striatum caused by the progressive loss of DA neurons in the substantia nigra pars compacta (SNpc), which is considered to be the primary cause of the motor symptoms of $\mathrm{PD}$, which include rigidity, bradykinesia, postural instability, gait disorder and tremor (2). In the last two decades, previous studies have employed animal models to gain an improved understanding of the PD process, as the availability of PD autopsy materials is limited. Although numerous animal models of PD have been employed in experimental studies concerning the mechanism of PD and therapeutic strategies (3-6), the unilateral 6-hydroxydopamine (6-OHDA) rat model, a neurotoxic compound-induced rat model possessing PD-associated pathological and behavioral features, is considered to be a classic animal model in PD correlation research.

The 6-OHDA rat model of PD was previously established by intracranial 6-OHDA injection into the SNpc, causing loss of tyrosine hydroxylase (TH)-positive neurons in the SNpc (4) and the TH-positive fibers in the striatum (5). It was also reported that death of $\mathrm{TH}^{+}$fibers in the striatum occurred prior to the $\mathrm{TH}^{+}$ neurons in the SNpc. This phenomenon seems to be a duplication of the human PD progress (6). Based on these studies, the unilateral PD rat model is often established by injection of 6-OHDA into one side of the striatum to reflect $\mathrm{TH}^{+}$neurons retrograde degeneration. Usually, the extent of the SNpc or striatal lesion is determined by examining turning behavior induced by amphetamine or apomorphine (APO). Although other behaviors, determined by rotarod, cylinder and open field tests, have been also observed in the model and used to determine the efficacy of drugs as potential PD therapeutics (6-8). However, there is 
a lack of evidence demonstrating a correlation between these non- amphetamine or -APO induced behaviors and DA neuron loss in the SNpc. Furthermore, different injection sites in the striatum have been employed in different studies, which makes interpreting behavioral and biochemical results from the rat model more difficult. Therefore, it is important to formulate a standard pattern of model establishment and phenotype analysis. In addition, systematic investigation of the associations between behavior, DA neuron loss and other pathological aspects during the course of PD in animal models should be performed, which may improve the evaluation of novel therapeutic approaches for PD.

In the present study, PD rat model was established by 6-OHDA unilateral injection in the neostriatal region of the striatum, and PD-associated behaviors and pathological features were detected. In addition, correlation analysis was performed between these phenotypes, and a potential intervention time-point, in addition to a comprehensive evaluation index system, was proposed for the assessment of PD therapeutic strategies using the PD rat model.

\section{Materials and methods}

Animals. A total of 60 male Sprague-Dawley rats (age, 7 weeks; weight, 220-240 g; Beijing Vital River Lab Animal Technology Co., Ltd., Beijing, China) were randomly assigned to the following two groups: Model $(n=38)$; and sham $(n=22)$. Rats were housed on a 12-h light/dark cycle with free access to food and water at $22-25^{\circ} \mathrm{C}$, relative humidity $40-60 \%$, and habituated to the housing conditions for 3 days prior to experiments. Every effort was made to minimize suffering and stress. All experimental procedures were approved by the Committee on Animal Care and Usage of Capital Medical University (Beijing, China).

Surgical procedure. Rats were anesthetized with an intraperitoneal injection of $6 \%$ chloral hydrate $(350 \mathrm{mg} / \mathrm{kg})$. Once anesthetized, rats were fixed in a stereotaxic apparatus (David Kopf Instruments, Tujunga, CA, USA) with a flat skull position. The skull was exposed and a $1 \mathrm{~mm}$ burr hole was drilled to detect the cranial cavity. The coordinates were as follows: Anteroposterior (AP), $0.8 \mathrm{~mm}$ from the bregma; mediolateral (ML), $2.7 \mathrm{~mm}$ from the midline; and dorsoventral (DV), -5.2 and -4.5 , respectively, from the skull (Fig. 1A) (9).

Subsequently, 6-OHDA [20 $\mu \mathrm{g}$ per rat in $4 \mu \mathrm{l}$ saline with $0.01 \%$ (w/v) ascorbic acid across two sites] was infused with an infusion pump through a $10 \mu$ l Hamilton syringe at a constant flow rate of $0.2 \mu \mathrm{l} / \mathrm{min}$ into the left striatum ( $2 \mu \mathrm{l}$ was injected at each coordinate). At the end of the infusion, the syringe was left implanted for an additional 5 min per site and was then slowly retracted. Sham-operated animals were submitted to the same procedure except $4 \mu \mathrm{l}$ vehicle $[0.9 \%$ saline containing $0.01 \%(\mathrm{w} / \mathrm{v})$ ascorbic acid] was infused into the striatum instead of 6-OHDA. During the surgery and recovery, animals were kept warm using a heating pad.

APO-induced rotations. APO-induced rotation was recorded using a multichannel rotometer system (RotoMax; AccuScan Instruments, Inc., Columbus, Ohio, USA), as described previously (10). Briefly, 2 weeks after surgery, all animals were injected subcutaneously with APO hydrochloride $(0.5 \mathrm{mg} / \mathrm{kg}$; Sigma-Aldrich; Merck KGaA, Darmstadt, Germany) and individually placed in the test cylinder. Rats that rotated in excess of 60 turns/30 min were considered to be the PD unilateral models. The drug-induced rotation was reexamined at $3,4,5$ and 6 weeks after surgery.

Rotarod test. The rotarod test was performed to evaluate motor coordination and balance, as described previously (11). Briefly, rats were trained to stay on the rotarod apparatus during a 5 min habituation trial $(10 \mathrm{rpm})$ of 2 consecutive days prior to the first testing day. Rats were subsequently subjected to a total of four rotarod test sessions with accelerating speeds (range, 4-40 rpm) over a period of 2 min on weeks $0,2,3,4,5$ and 6 after surgery. Each test session was composed of two trials on the rotarod, with a maximum duration of $2 \mathrm{~min}$ per trial and a 20 min inter-trial interval. The time duration of each animal staying on the rod was recorded as the latency to fall, which was registered automatically by a trip switch under the floor of each rotating drum. The best score achieved by each rat was used for further analysis.

Cylinder test. Because unilateral injection of 6-OHDA can cause limb impairment, the cylinder test was performed to investigate spontaneous forelimb lateralization, taking advantage of the natural exploratory instinct of rodents to a new environment (12). The test was performed as follows: Rats were placed individually inside a glass cylinder (diameter, $22 \mathrm{~cm}$; height, $26 \mathrm{~cm}$ ) with two mirrors located behind the cylinder at a $45^{\circ}$ angle to allow $360^{\circ}$ vision. The rats were video recorded for 5 min after rats first touched the walls of the cylinder with the impaired or unimpaired forelimb or both simultaneously. Each individual rearing episode was counted by a blinded researcher. The scores were calculated by the following asymmetry ratio: Left-right/(right + left + both). Scores on the forelimb asymmetry ratio range from -1 to 1 . The positive ratio was consistent with greater use of the unimpaired forelimb over the impaired forelimb. By contrast, the negative asymmetry ratio suggests greater use of the impaired forelimb compared with the unimpaired forelimb. Therefore, a high positive ratio would be consistent with a hemiparkinsonian lesion.

Open field test. Open field tests were performed at 0, 2, 3, 4, 5 and 6 weeks after surgery, as described previously (13). Briefly, all behavioral procedures were performed between 9:00 am and 3:00 pm, and silence in the room was maintained for the duration of the test. Locomotor activity was measured in automated activity chambers connected to an analyzer that transmitted the number of beam breaks (activity data) to a computer (VersaMon version 2.01; Accuscan Instruments, Inc.). The rats were placed individually in the center of the chamber. Each chamber consisted of an individual cage with a grid of infrared beams mounted horizontally every $2.5 \mathrm{~cm}$. Locomotor activity was quantified as the number of beam interruptions (crossings) registered by a computer and recorded as total movement distance $(\mathrm{cm})$, velocity $(\mathrm{cm} / \mathrm{sec})$, rest time (sec), center entries (times) and moves (times) during the $30 \mathrm{~min}$ recording period. The open field chambers were washed with $75 \%$ ethanol solution each time prior to behavioral testing in order to eliminate odors left by the previous rat. 
Immunohistochemistry (IHC). Following the behavioral tests each week, 10 animals ( $n=6$ for model and $n=4$ for sham) were anesthetized with $6 \%$ chloral hydrate and perfused intracardially with warm $0.9 \% \mathrm{NaCl}$ at room temperature, followed by $200 \mathrm{ml}$ cold $4 \%$ paraformaldehyde (PFA/0.1 M PBS). The brains were rapidly removed from the skull following decapitation and immersed in $4 \%$ paraformaldehyde for $24 \mathrm{~h}$ at $4^{\circ} \mathrm{C}$. Subsequently, the tissues were cryoprotected with 20 and $30 \%$ sucrose sequentially, until they sank to the bottom of the tube. Brains were subsequently embedded in optimal cutting temperature medium at $-80^{\circ} \mathrm{C}$ overnight. The coronal brain sections were obtained using a microtome at $30 \mu \mathrm{m}$ thickness for the striatum and $50 \mu \mathrm{m}$ thickness for the midbrain including the SNpc. IHC was performed on free-floating sections, which were rinsed in 0.1 M PBS three times for $5 \mathrm{~min}$. Sections were then permeabilized with $0.3 \%$ Triton-X-100 for $30 \mathrm{~min}$ at room temperature and washed with $0.1 \mathrm{M}$ PBS three times for $5 \mathrm{~min}$. Sections were treated with $3 \%$ hydrogen peroxide for $30 \mathrm{~min}$ and washed with $0.1 \mathrm{M}$ PBS three times for $5 \mathrm{~min}$. Subsequently, sections were blocked with $5 \%$ normal goat serum (Vector Laboratories, Inc., Burlingame, CA, USA) in 0.1 M PBS for $1 \mathrm{~h}$ at room temperature, which was followed by incubation with primary antibodies overnight at $4^{\circ} \mathrm{C}$. The following primary antibodies were used in the present study: Anti-TH (mouse; 1:5,000; cat. no. T1299; Sigma-Aldrich; Merck KGaA; USA); anti-glial fibrillary acidic protein (GFAP; mouse; 1:500; cat. no. MAB360; EMD Millipore, Billerica, MA, USA); and anti-CD11b (Mac1; mouse; 1:500; cat. no. MCA275G; Bio-Rad Laboratories, Inc., Hercules, CA, USA). All antibodies were diluted in $5 \mathrm{ml} 0.1 \mathrm{M}$ PBS. Staining was performed using Vectastain Universal Elite ABC kit (Vector Laboratories, Inc. USA). Biotinylated anti-mouse IgG secondary antibodies (1:200) were used to recognize primary antibodies at $37^{\circ} \mathrm{C}$ for $60 \mathrm{~min}$, followed by washing three times and incubation with a streptavidin-horseradish peroxidase complex $(1: 1,000)$ at $37^{\circ} \mathrm{C}$ for $30 \mathrm{~min}$. The immunoreactivities were visualized by 3,3-diaminobenzidine (DAB) within $2 \mathrm{~min}$. The sections were mounted, coverslipped and dehydrated in a gradual concentration of ethanol.

The number of TH-positive cells in the SNpc was determined by stereological measurements using the optical fractionator method in a computerized system (Stereo Investigator software, version 8.0; Leica Microsystems $\mathrm{GmbH}$, Wetzlar, Germany), as previously described $(14,15)$. The sections were used for counting, including the entire SNpc from the rostral tip of the pars compacta back to the caudal end of the pars reticulate. Every eighth section throughout the entire SNpc was counted, with a total of 6 sections for each animal. The estimates of the total number of neurons were calculated according to the optical fractionator formula, and coefficients of error $<0.10$ were accepted. Both the injected and non-injected side of the SNpc was quantified.

For measurement of TH fibers in the striatum, briefly, high-resolution images were obtained from the sections stained by TH using a x1.25 (objective lens) Nikon light microscope. The extent of striatal denervation was measured in three sections per animal corresponding to $+1.2,+0.6$ and $-0.2 \mathrm{~mm}$ from the bregma, using Image-Pro Plus 6.0 (Media Cybernetics, Inc., Rockville, MD, USA). The entire striatum according to the dorsoventral axis was divided into equal halves and the measured values were corrected for nonspecific background staining by subtracting values of the blank area. Data are presented as the percentage of striatal densitometry with the non-injected hemisphere corresponding to $100 \%$ for each individual rat.

The area occupied by Mac1- or GFAP-positive staining was defined by densitometry using Image-Pro Plus 6.0. All the analyses were performed by an investigator blind to different samples. Data were normalized to the contralateral normal side.

Statistical analysis. Data was analyzed with SPSS 21.0 software (IBM Corp., Armonk, NY, USA) and presented as the mean \pm standard error of the mean. Differences between the means of two groups were analyzed using independent-samples Student's t-test or, when data were not normally distributed, a nonparametric Mann-Whitney U test was performed. For correlation analysis, the Pearson's correlation coefficient, and subsequent linear regression, was determined. $\mathrm{P}<0.05$ was considered to indicate a statistically significant difference.

\section{Results}

The behavior of $P D$ rats. In the present study, the neostriatum was selected as the injection site, which is illustrated in Fig. 1A. (AP=0.8 mm, ML=2.7 mm and DV=-5.2 and $-4.5 \mathrm{~mm}$ ). The experiment was performed according to the schedule as presented in Fig. 1A. At 2 weeks after surgery, the rats with $>60$ rotations $/ 30$ min induced by APO were considered successful PD models. According to the criteria, a total of 30 PD model rats were obtained.

In order to characterize the behaviors of the hemiparkinsonian rat during the course of dopaminergic nigrostriatal pathway degeneration, the current study observed several behavioral parameters, including APO-induced rotation, forelimb placement, motor coordination and locomotor activity from weeks 2 to 6 following striatum injection. It was demonstrated in Fig. 1B that, in the model group, the number of APO-induced contralateral rotations during a $30 \mathrm{~min}$ testing period was increased following surgery between weeks $2(69.3 \pm 10.1 \mathrm{turns} / 30 \mathrm{~min})$ and $6(211.6 \pm 27.2 \mathrm{turns} / 30 \mathrm{~min})$. Strikingly, the rotations sharply increased from week 2 $(69.3 \pm 10.1$ turns $/ 30 \mathrm{~min})$ to week $3(153.0 \pm 15.5$ turns/30 min), after which the number of turns remained quite stable at around 200 turns/30 min. The number of turns/30 min did not change over the course of this experiment in sham rats.

The motor coordination and balance skills in the PD rats were assessed by the rotarod test. The mean time the model group stayed on the accelerating rotarod was significantly shorter compared with the sham group $(\mathrm{P}<0.01$; Fig. $1 \mathrm{C})$ between weeks 2 and 6 after surgery. Notably, the latency to fall in the rotarod test was decreased gradually across the whole experimental period in the model group (week 2, $47.2 \pm 2.8 \mathrm{sec}$; week $6,41.2 \pm 2.9 \mathrm{sec}$ ).

To appraise the forelimb placement of PD rats, the cylinder test was performed. The ratio of forelimb placement [(left-right)/(right + left + both)] of the model group was significantly higher compared with the sham group $(\mathrm{P}<0.01$; Fig. 1D) during the experimental period.

In addition, the open field test was performed to examine locomotor activity and anxiety-associated behaviors (8). 
A
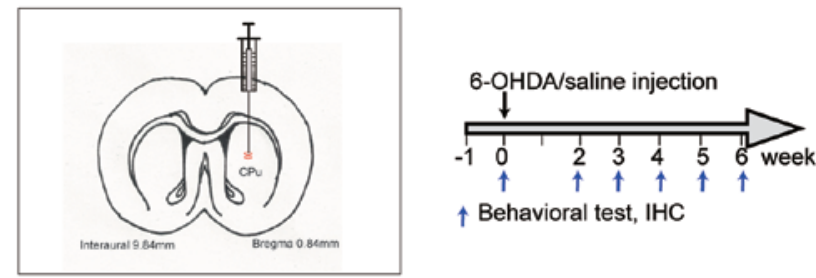

B
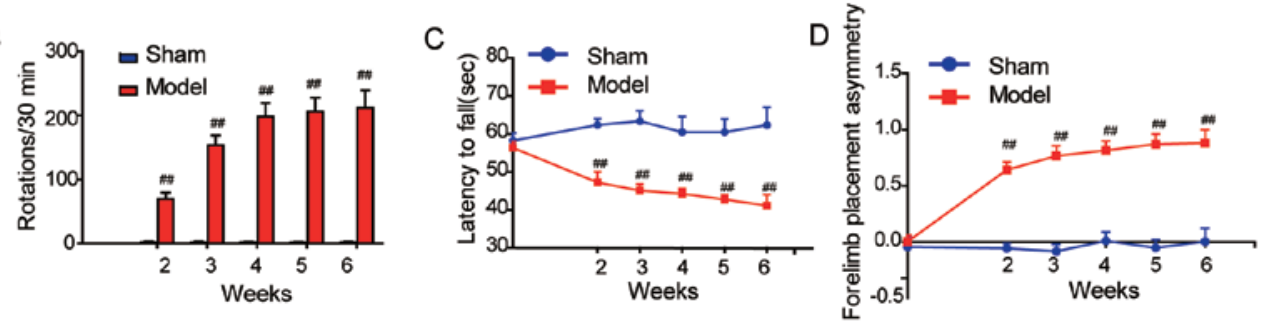

E
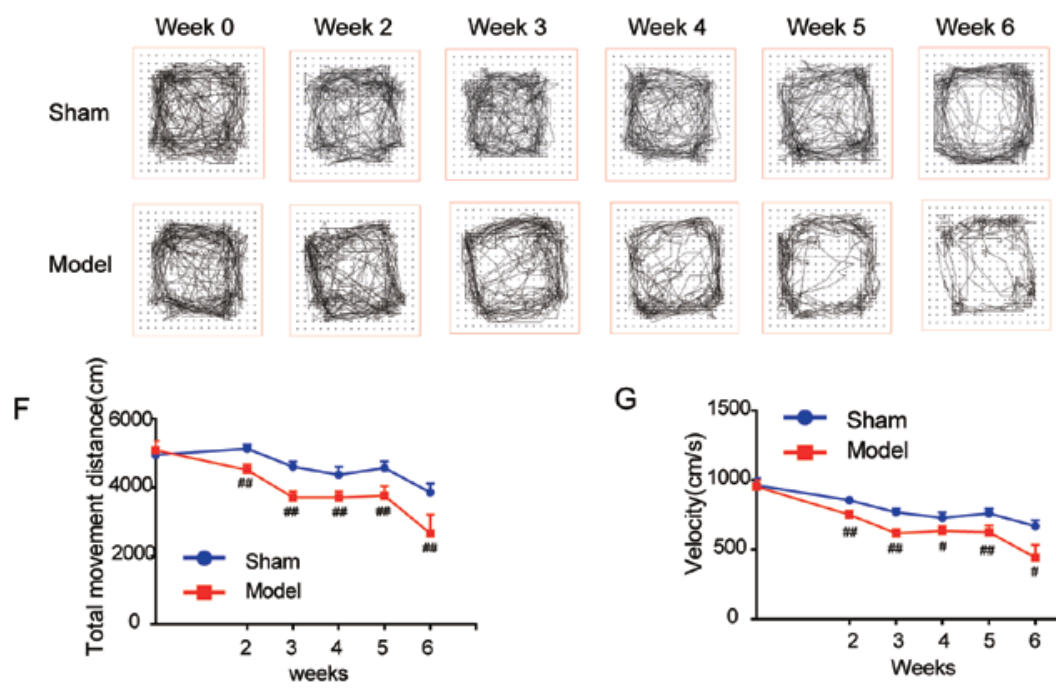

G
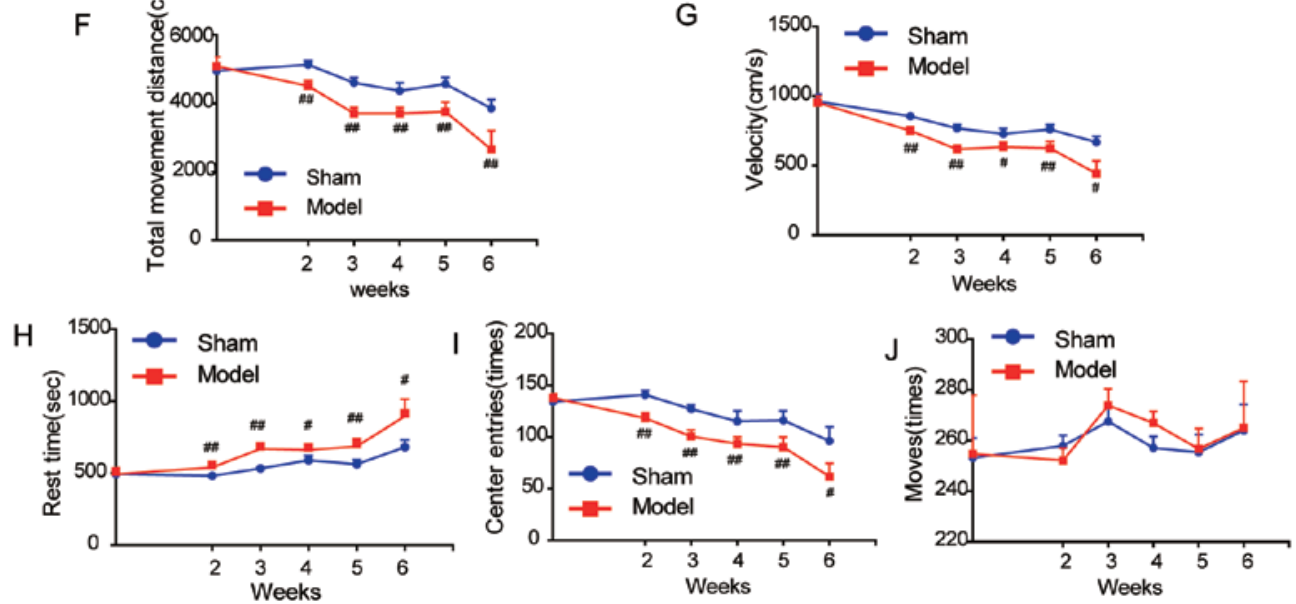

Figure 1. Behavioral performance of the 6-OHDA rat model of Parkinson's disease. (A) Stereotaxic coordinates of the left striatum (anteroposterior=0.8 mm, mediolateral $=2.7 \mathrm{~mm}$ and dorsoventral=-5.2 and $-4.5 \mathrm{~mm}$ ) and the study time-line. Behavior of rats was evaluated by (B) apomorphine-induced rotation, (C) rotarod and (D) cylinder tests. (E) Representative graphs from open field test. (F) Total movement distance, (G) velocity, (H) rest time, (I) center entries and (J) moves were recorded during the open field tests. Data are presented as the mean + standard error of the mean. Week $0, n=38$ and $n=22$ in model and sham groups, respectively; week $2, n=30$ and $n=22$ in model and sham groups, respectively; week $3, n=24$ and $n=18$ in model and sham groups, respectively; week $4, n=18$ and $n=14$ in model and sham groups, respectively; week 5, n=12 and n=10 in model and sham groups, respectively; week $6, n=6$ and $n=6$ in model and sham groups respectively. ${ }^{\#} \mathrm{P}<0.05 ;{ }^{\# \#} \mathrm{P}<0.01$ vs. sham. 6-OHDA, 6-hydroxydopamine; IHC, immunohistochemistry.

Several parameters were determined in the test, and significant decreases in total movement distance $(\mathrm{cm})$, velocity $(\mathrm{cm} / \mathrm{sec})$, center entries (times), and an increase in rest time (sec), were observed in the model group compared with the sham group $(\mathrm{P}<0.01$; Fig. 1E-I). However, no significant differences in the moves (times) between the model and sham group were observed (Fig. 1J; P>0.05).

IHC staining. TH is the rate-limiting enzyme in DA synthesis and an established marker for DA neurons. IHC was used to determine the effects of 6-OHDA on $\mathrm{TH}^{+}$neurons and fibers (Fig. 2). To confirm DA neuron deficiency, stereological quantification of $\mathrm{TH}^{+}$neurons in the $\mathrm{SNpc}$ was performed in all groups at weeks 2-6 following surgery. As anticipated, the number of surviving $\mathrm{TH}^{+}$neurons in the $\mathrm{SNpc}$ was significantly decreased in $\mathrm{PD}$ rats compared with sham rats $(\mathrm{P}<0.01$; Fig. 2A and $\mathrm{C}$ ). Furthermore, the survival of $\mathrm{TH}^{+}$neurons (from $47 \%$ in week 2 to $20 \%$ in week 6 ) in the model group in SNpc descended progressively across the experimental period. Consistently, $\mathrm{TH}^{+}$fiber density in the striatum of the model 

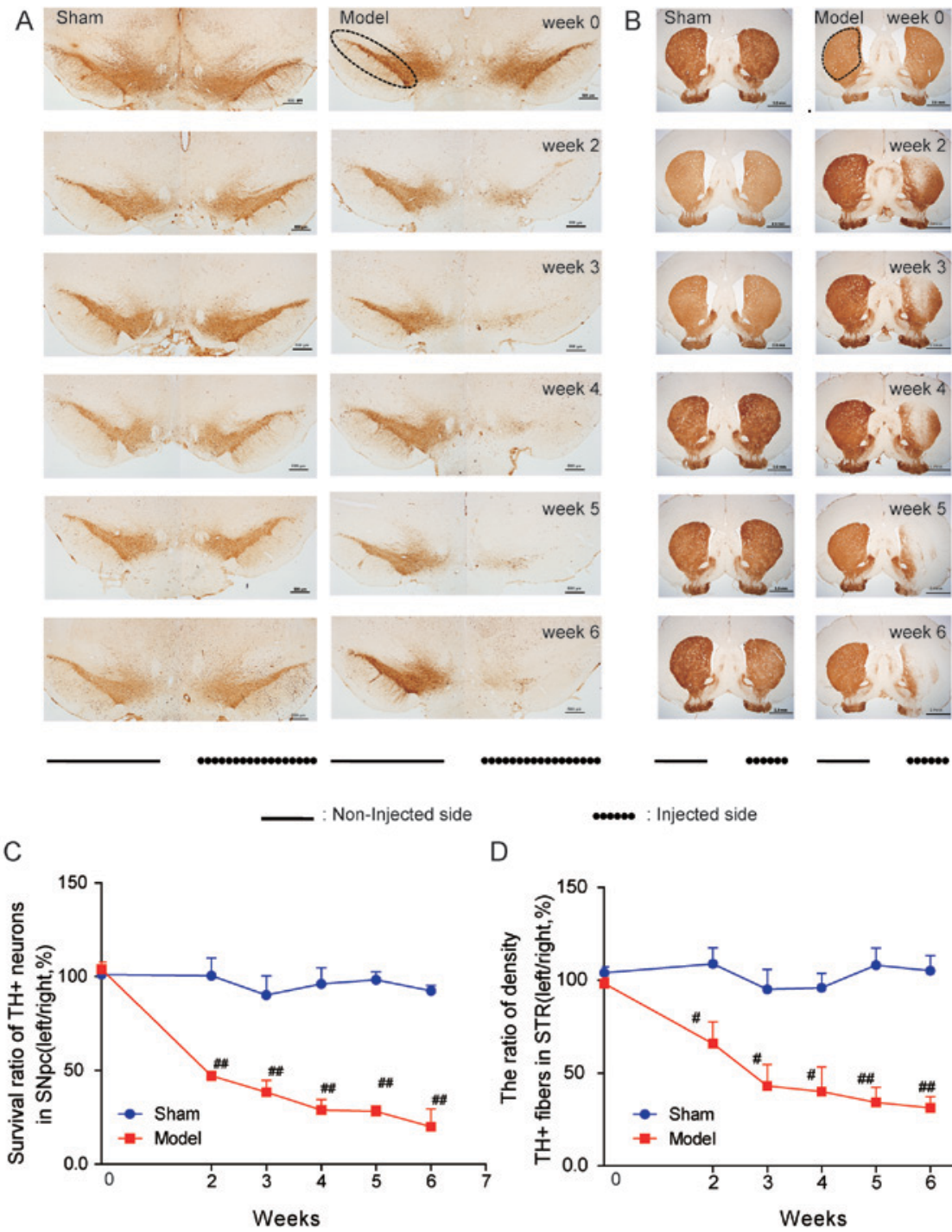

week 2

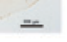

week 3
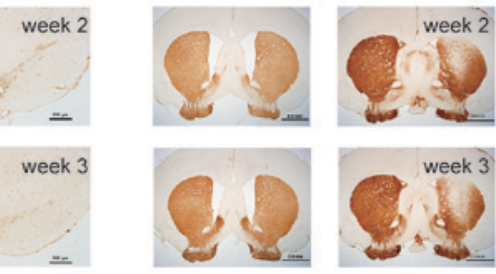

week 4 .
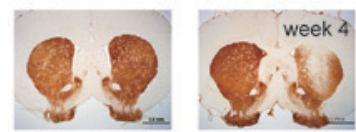

week 5
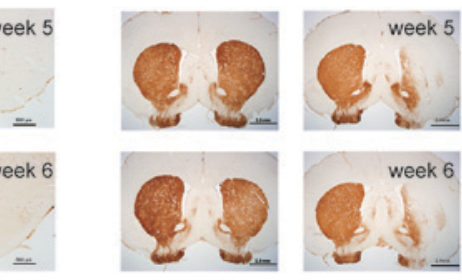

c

D

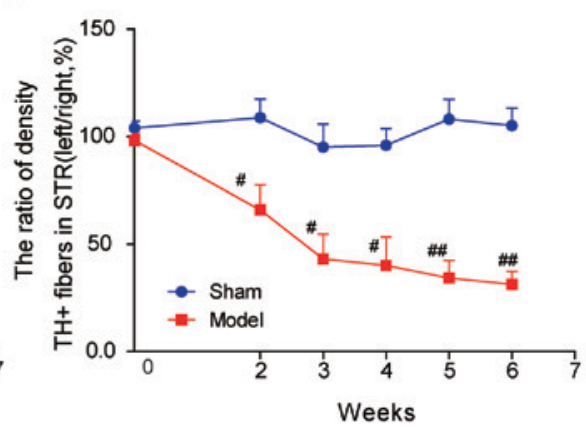

Figure 2. Parkinson's disease-associated pathology in the 6-OHDA rat model. (A) IHC staining for $\mathrm{TH}^{+}$neurons in the $\mathrm{SNpc}$. Scale bar=500 $\mu \mathrm{m}$. (B) IHC staining for $\mathrm{TH}^{+}$fibers in the STR following 6-OHDA or saline injection in the left STR. Scale bar=2 mm. (C) Ratio (left/right) of stereological quantification of numbers of $\mathrm{TH}^{+}$neurons in the SNpc. (D) Ratio (left/right) of $\mathrm{TH}^{+}$fiber density in the STR. Data are presented as the mean + standard error of the mean. $\mathrm{n}=4$ in sham group and $\mathrm{n}=6$ in model group at each time-point. ${ }^{\#} \mathrm{P}<0.05$ and ${ }^{\#} \mathrm{P}<0.01$ vs. sham. 6-OHDA, 6-hydroxydopamine; IHC, immunohistochemistry; TH, tyrosine hydroxylase; SNpc, substantia nigra pars compacta; STR, striatum.

group was lower compared with the sham group $(\mathrm{P}<0.01$; Fig. 2B and D).

As glial reactivity is considered to be a crucial event in the process of 6-OHDA toxicity in the PD model (16), the present study also observed the morphological alterations of glial cells activated by 6-OHDA (Fig. 3). The population of astrocytes and microglia in the rat brain were presented as the percentage of area occupied by $\mathrm{GFAP}^{+}$(specific for astrocyte) and $\mathrm{Macl}^{+}$ (specific for microglia) cells in the ipsilateral SNpc, respectively, at all time-points. There was a significant increase in the area occupied by $\mathrm{GFAP}^{+}$cells in the $\mathrm{SNpc}$ of the model group compared with the sham group $(\mathrm{P}<0.01$; Fig. $3 \mathrm{~A}$ and $\mathrm{C})$ at weeks 3, 5, 6. The area occupied with $\mathrm{Macl}^{+}$cells began to increase at week 2 in the SNpc of the model group compared with the sham group, and the microglial activation persisted at weeks 3, 4, 5 and 6 ( $\mathrm{P}<0.01$; Fig. 3B and $\mathrm{D})$.

Correlation between morphological and behavioral measures. In order to investigate the association between the survival of nigral DA neurons and these behavioral tests in the PD rat model, linear regression analysis was performed. The survival of nigral DA neurons within the SNpc was correlated with APO-induced rotations $\left(\mathrm{R}^{2}=0.430, \mathrm{P}=0.001\right.$; Fig. $\left.4 \mathrm{~A}\right)$, but not correlated with either the area of GFAP ${ }^{+}$staining $\left(\mathrm{R}^{2}=0.066\right.$, $\mathrm{P}=0.169$; Fig. 4B) or the area of $\mathrm{Macl}^{+}$staining $\left(\mathrm{R}^{2}=0.018\right.$, $\mathrm{P}=0.477$; Fig. 4C) in the SNpc. The latency to fall of the rotarod test $\left(\mathrm{R}^{2}=0.163, \mathrm{P}=0.027\right.$; Fig. $\left.4 \mathrm{D}\right)$ and the paw preference (asymmetry) in the cylinder test $\left(\mathrm{R}^{2}=0.279, \mathrm{P}=0.003\right.$; Fig. $4 \mathrm{E}$ ) were strongly correlated with the survival of DA neurons in the SNpc, indicating that these two behavioral tests together with APO-induced rotations may be an applicable evaluation index for PD-associated behaviors induced by 6-OHDA.

\section{Discussion}

The customary model of PD may be established by the injection of 6-OHDA into one of the following three targets: $\mathrm{SNpc}$, medial forebrain bundle or the neostriatal region. The 
A
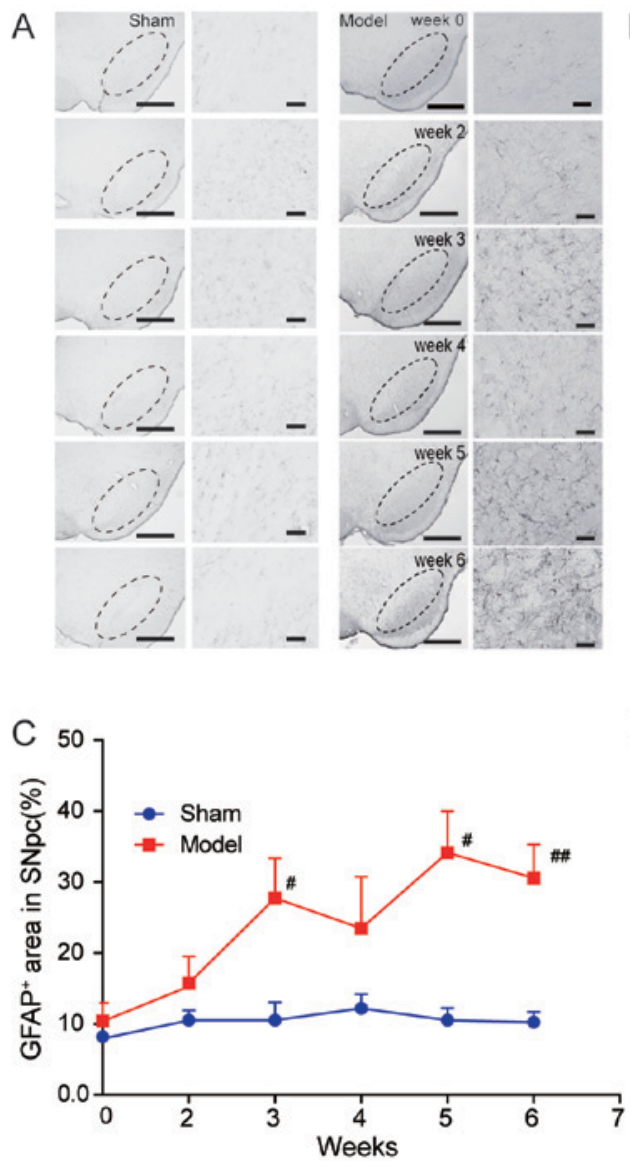

B
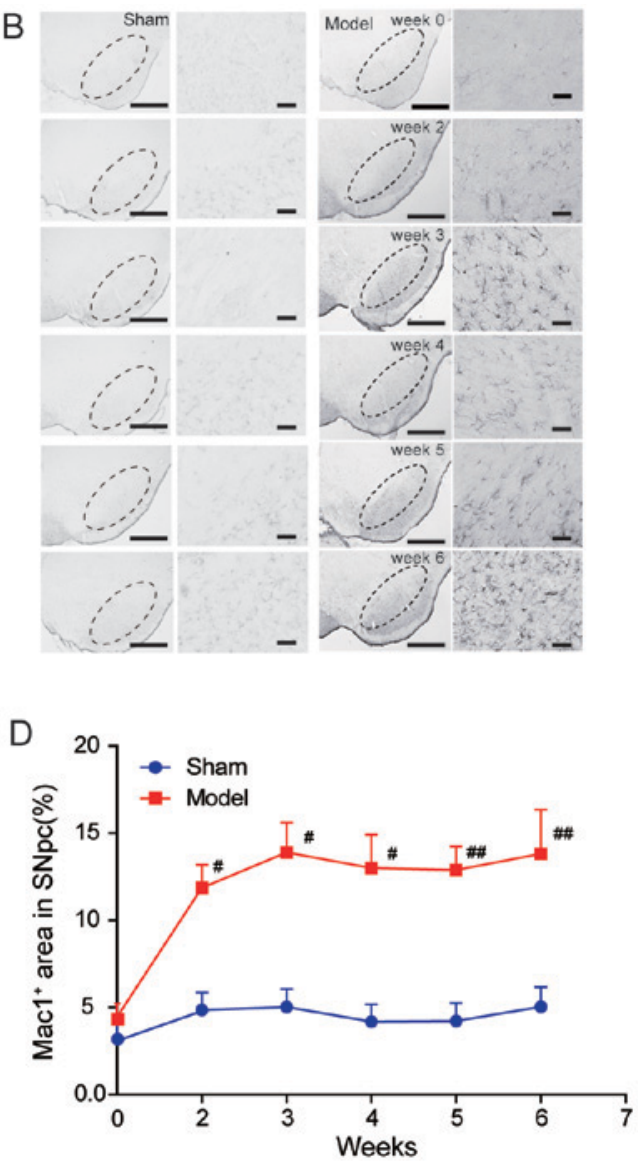

Figure 3. The glial response in the Parkinson's disease rat model. (A) Representative IHC staining for GFAP ${ }^{+}$astrocytes and quantified percentage of area occupied by GFAP ${ }^{+}$cells. (B) Mac1 ${ }^{+}$microglia IHC staining in SNpc and quantified percentage of area occupied by Mac1 ${ }^{+}$cells. Scale bar=1 mm in the left row, Scale bar $=50 \mu \mathrm{m}$ in the right row. Quantified percentage area occupied by (C) GFAP ${ }^{+}$and (D) Mac1 ${ }^{+}$cells. Data are presented as the mean + standard error of the mean. $\mathrm{n}=4$ in sham group and $\mathrm{n}=6$ in model group at each time-point. ${ }^{\#} \mathrm{P}<0.05$ and ${ }^{\# \#} \mathrm{P}<0.01$ vs. sham. IHC, immunohistochemistry; GFAP, glial fibrillary acidic protein; Mac1, CD11b; SNpc, substantia nigra pars compacta.

latter is established to be an appropriate site for establishment of a slow progressive PD animal model (7). Although the 6-OHDA lesion PD rat or mouse model has been widely used for decades (17-20), the present study, to the best of our knowledge, was the first to demonstrate, over a long time period, the correlation between progressive nigrostriatal neurodegeneration and the degree of APO-induced and non-drug-induced behavioral impairments, however, no correlation was observed with morphological glial reactivity in the SNpc of the rat model. The present study indicates relatively stable time-points for therapeutic interventions and also provides a system for evaluation of the PD rat model even when APO is not available.

In the current study, the injection site of the striatum was selected as it is the neostriatum area in the rat that is the specific affected field in the human brain that results in the PD-associated motor deficits and the progression of PD pathology (7). It is widely accepted that with the neurotoxin-induced neuronal degeneration, the DA receptors on the injected side of the striatum become relatively hypersensitive to stimulation by its ligands. APO, a DA agonist, stimulates the receptors causing a higher activity in the striatum on the injected side relative to the non-injected side, ultimately inducing a rotation of the body turning contralateral from the lesioned side (7). The PD model is well-recognized by the characteristic rotational behavior of rodents administrated with dopaminomimetics. This drug-induced rotational behavior is associated with the degree of the nigrostriatal $\mathrm{TH}^{+}$protein loss (7). Thus, the APO-induced rotations (60 turns/30 min) is considered the criterion to identify the rat model of PD in this experiment.

In addition, to thoroughly assess the 6-OHDA rat model of PD, other non-drug-induced behavioral tests, including cylinder, rotarod and open field tests, were investigated as symptomatic parkinsonian signs of the rat model. The number of APO-induced contralateral rotations during a 30 min testing period sharply increased between weeks 2 and 3 following surgery, whereas rotation number remained stable between weeks 4 and 6 . This phenomenon was consistent with the results of cylinder and rotarod tests (Fig. 1). Previous studies have demonstrated that 6-OHDA injection into the striatum led to a protracted retrograde DA neuron-specific degeneration, which usually occurs after $1-3$ weeks $(10,21-23)$. In the present study, the survival of DA neurons in the SNpc of the rat model decreased progressively (Fig. 2). When linear regression analysis was performed, APO-induced rotations exhibited a negative correlation with the survival of DA neurons, which was also positively correlated with latency to fall from rotarod and negatively correlated with paw preference (asymmetry) on the cylinder test. Therefore, we hypothesized that the 6-OHDA model may be evaluated by one or all of the tests that exhibited 

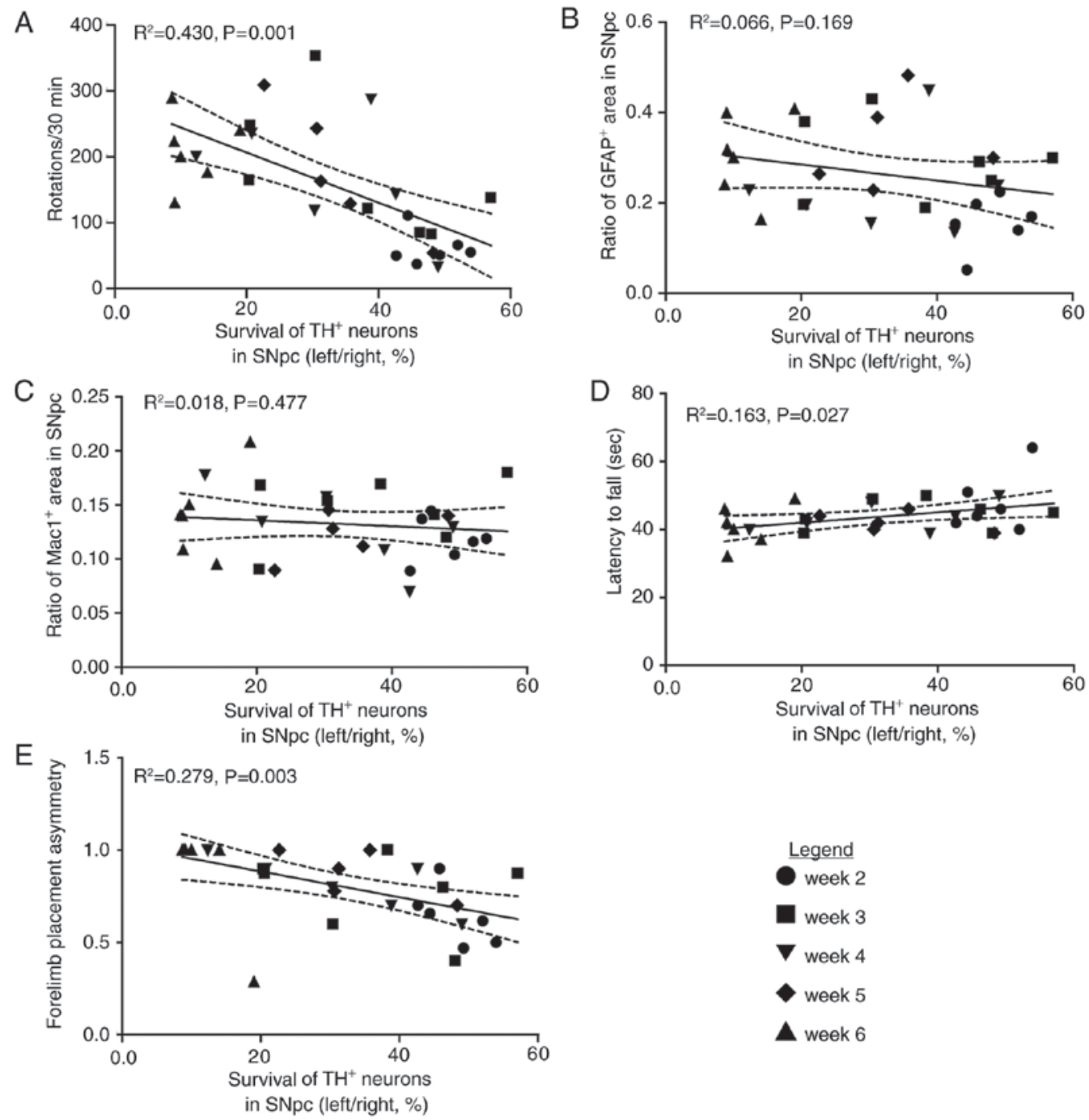

in SNpc (left/right, \%)

Figure 4. The correlation between dopamine neuron loss and glial activation, in addition to behavioral performances. (A) Apomorphine-induced rotations were negatively correlated with the survival of $\mathrm{TH}^{+}$neurons in the $\mathrm{SNpc}$. The survival of $\mathrm{TH}^{+}$neurons was not correlated with the ratio of $(\mathrm{B}) \mathrm{GFAP}^{+}$area or (C) $\mathrm{Macl}^{+}$area in the SNpc. (D) Latency to fall was positively correlated with the survival of $\mathrm{TH}^{+}$neurons in the $\mathrm{SNpc}$. (E) Forelimb placement asymmetry was negatively correlated with the survival of $\mathrm{TH}^{+}$neurons in the $\mathrm{SNp}$. The fitted line is framed within the $95 \%$ confidence intervals indicated by dashed lines. TH, tyrosine hydroxylase; SNpc, substantia nigra pars compacta; GFAP, glial fibrillary acidic protein; Mac1, CD11b.

a significant correlation with the survival of DA neurons, according to experimental conditions.

For the open field test, several parameters, including total movement distance $(\mathrm{cm})$, velocity $(\mathrm{cm} / \mathrm{sec})$, rest time $(\mathrm{sec})$, moves (times) and center entries (times), were observed as these parameters were reported to represent the locomotor activity and anxiety behaviors of PD rats $(7,24)$. Locomotor activity has also been reported to be associated with alterations in DA transmitter function, for example, decreased DA levels may lead to reduced locomotion (7). In the present study, a reduced level of locomotor activity was observed in the PD rats. Therefore, it is clear that the spontaneous movements associated with alterations in DA transmitter function of PD rats may be estimated using the open field test.

In terms of the association between PD-associated pathology and behavior, the results indicate that the extent of nigral DA neuron loss in the SNpc may be predicted through both drug-induced rotations and non-drug-induced movement behaviors. Consistently, the $\mathrm{TH}^{+}$fiber density in the striatum of the PD rat model also decreased in a slow progressive pattern (Fig. 2). Consistent with the results of other studies that employed injection of 6-OHDA into the striatum, where striatal terminal damage was observed within 1 day of injection and SNpc neuron loss was minimal until 1 week later, reaching a maximum within 2-3 weeks $(10,21,25)$, the present study demonstrated a slowly developing partial lesion of the nigrostriatal pathway induced by 6-OHDA. Compared with other studies $(18,25)$, a little difference in the time-point of the lesion of nigral neuron was observed, i.e., it exhibited swift changes from week 2 to week 3 maintained kept a stable state from week 3 to week 6 . The detectable discrepancy may result from diverse injection sites in the striatum.

Glial responses are considered to exhibit dual effects in the process of neurodegeneration. The activation of glial cells may benefit DA neurons at early stages of PD, however, the excessive continued activation of glia was reported to be harmful to the neurons $(16,26,27)$. In the present study, the activation of astrocytes and microglia was observed throughout the impairment of the nigrostriatal pathway in the SNpc (Fig. 3). Notably, although the continued lesion of DA neurons was observed 
during the period of observation, the survival of $\mathrm{TH}^{+}$neurons within the SNpc was not correlated with the glial activation in the SNpc. Considering the crucial role of glial responses, particularly microglial activation in PD pathogenesis (16), the correlation data in the present study indicate that the abnormal activation of glia cells was not linked with loss of dopaminergic neurons induced by 6-OHDA, and other pathways such as cytokines or inflammatory factors may also be involved in the process, which may not be associated with morphological microglial activation (28).

In conclusion, it is of great importance to formulate a standard index of key characteristics of the 6-OHDA lesion PD model for assessing novel drugs, developing novel treatment strategies and understanding the nature of the pathogenic processes of PD. The results of the current study may be useful in appraising the potential of novel treatment strategies for PD and in investigating the mechanisms associated with functional recovery of dopaminergic neurons in the PD brain.

\section{Acknowledgements}

The present study was supported by the Chinese National Basic Research Program (grant no. 2011CB504100), the Important National Science and Technology Specific Project (grant no. 2011ZX09102-003-01) and the Projects Under Beijing Municipality (grant no. IDHT20140514). The authors thank Ms Min Sun and Ms Xiaoli Gong (Department of Neurobiology, Capital Medical University, Beijing, China) for their technical support.

\section{References}

1. Redgrave P, Vautrelle N and Reynolds JN: Functional properties of the basal ganglia's re-entrant loop architecture: Selection and reinforcement. Neuroscience 198: 138-151, 2011.

2. Su X and Federoff HJ: Immune responses in Parkinson's disease: Interplay between central and peripheral immune systems. Biomed Res Int 2014: 275178, 2014

3. Jackson-Lewis V, Blesa J and Przedborski S: Animal models of Parkinson's disease. Parkinsonism Relat Disord 18 (Suppl 1): S183-S185, 2012.

4. Ungerstedt U: 6-Hydroxy-dopamine induced degeneration of central monoamine neurons. Eur J Pharmacol 5: 107-110, 1968.

5. Blandini F, Armentero MT and Martignoni E: The 6-hydroxydopamine model: News from the past. Parkinsonism Relat Disord 14 (Suppl 2): S124-S129, 2008.

6. Lee CS, Sauer H and Bjorklund A: Dopaminergic neuronal degeneration and motor impairments following axon terminal lesion by instrastriatal 6 -hydroxydopamine in the rat. Neuroscience 72 : 641-653, 1996.

7. Bové J and Perier C: Neurotoxin-based models of Parkinson's disease. Neuroscience 211: 51-76, 2012.

8. Meredith GE and Kang UJ: Behavioral models of Parkinson's disease in rodents: A new look at an old problem. Mov Disord 21: 1595-1606, 2006.

9. Paxinos G and Watson C: The Rat Brain in Stereotaxic Coordinates. Academic Press, 2005.

10. Przedborski S, Levivier M, Jiang H, Ferreira M, Jacksonlewis V, Donaldson D and Togasaki DM: Dose-dependent lesions of the dopaminergic nigrostriatal pathway induced by intrastriatal injection of 6-hydroxydopamine. Neuroscience 67: 631-647, 1995.

11. Monville C, Torres EM and Dunnett SB: Comparison of incremental and accelerating protocols of the rotarod test for the assessment of motor deficits in the 6-OHDA model. J Neurosci Methods 158: 219-223, 2006.
12. Landers MR, Kinney JW and van Breukelen F: Forced exercise before or after induction of 6-OHDA-mediated nigrostriatal insult does not mitigate behavioral asymmetry in a hemiparkinsonian rat model. Brain Res 1543: 263-270, 2014.

13. Yuan HL, Li B, Xu J, Wang Y, He Y, Zheng Y and Wang XM: Tenuigenin protects dopaminergic neurons from inflammation-mediated damage induced by the lipopolysaccharide. CNS Neurosci Ther 18: 584-590, 2012.

14. Van der Perren A, Macchi F, Toelen J, Carlon MS, Maris M, de Loor H, Kuypers DR, Gijsbers R, Van den Haute C, Debyser Z and Baekelandt V: FK506 reduces neuroinflammation and dopaminergic neurodegeneration in an $\alpha$-synuclein-based rat model for Parkinson's disease. Neurobiol Aging 36: 1559-1568, 2015.

15. Sanchez-Guajardo V, Febbraro F, Kirik D and Romero-Ramos M: Microglia acquire distinct activation profiles depending on the degree of alpha-synuclein neuropathology in a rAAV based model of Parkinson's disease. PLoS One 5: e8784, 2010.

16. McGeer PL and McGeer EG: Glial reactions in Parkinson's disease. Mov Disord 23: 474-483, 2008.

17. Mendez JS and Finn BW: Use of 6-hydroxydopamine to create lesions in catecholamine neurons in rats. Neurosurg 42: 166-173, 1975.

18. Stott SR and Barker RA: Time course of dopamine neuron loss and glial response in the 6-OHDA striatal mouse model of Parkinson's disease. Eur J Neurosci 39: 1042-1056, 2014.

19. Schlachetzki JC, Marxreiter F, Regensburger M, Kulinich A, Winner B and Winkler J: Increased tyrosine hydroxylase expression accompanied by glial changes within the non-lesioned hemisphere in the 6-hydroxydopamine model of Parkinson's disease. Restor Neurol Neurosci 32: 447-462, 2014.

20. Walsh S, Finn DP and Dowd E: Time-course of nigrostriatal neurodegeneration and neuroinflammation in the 6-hydroxydopamine-induced axonal and terminal lesion models of Parkinson's disease in the rat. Neuroscience 175: 251-261, 2011.

21. Sauer H and Oertel WH: Progressive degeneration of nigrostriatal dopamine neurons following intrastriatal terminal lesions with 6-hydroxydopamine: A combined retrograde tracing and immunocytochemical study in the rat. Neuroscience 59: 401-415, 1994.

22. Marti MJ, Saura J, Burke RE, Jackson-Lewis V, Jiménez A, Bonastre $\mathrm{M}$ and Tolosa E: Striatal 6-hydroxydopamine induces apoptosis of nigral neurons in the adult rat. Brain Res 958: 185-191, 2002.

23. Marti MJ, James CJ, Oo TF, Kelly WJ and Burke RE: Early developmental destruction of terminals in the striatal target induces apoptosis in dopamine neurons of the substantia nigra. J Neurosci 17: 2030-2039, 1997.

24. Prut $L$ and Belzung C: The open field as a paradigm to measure the effects of drugs on anxiety-like behaviors: A review. Eur J Pharmacol 463: 3-33, 2003.

25. Blandini F, Levandis G, Bazzini E, Nappi G and Armentero MT: Time-course of nigrostriatal damage, basal ganglia metabolic changes and behavioural alterations following intrastriatal injection of 6-hydroxydopamine in the rat: New clues from an old model. Eur J Neurosci 25: 397-405, 2007.

26. Mosley RL, Benner EJ, Kadiu I, Thomas M, Boska MD, Hasan K, Laurie $\mathrm{C}$ and Gendelman HE: Neuroinflammation, oxidative stress and the pathogenesis of Parkinson's disease. Clin Neurosci Res 6: 261-281, 2006.

27. Vivekanantham S, Shah S, Dewji R, Dewji A, Khatri C and Ologunde R: Neuroinflammation in Parkinson's disease: Role in neurodegeneration and tissue repair. Int J Neurosci 125: 717-725, 2015.

28. Depino AM, Earl C, Kaczmarczyk E, Ferrari C, Besedovsky H, del Rey A, Pitossi FJ and Oertel WH: Microglial activation with atypical proinflammatory cytokine expression in a rat model of Parkinson's disease. Eur J Neurosci 18: 2731-2742, 2003.

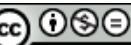

This work is licensed under a Creative Commons Attribution-NonCommercial-NoDerivatives 4.0 International (CC BY-NC-ND 4.0) License. 